\title{
COMMUNICATION
}

\section{Prise en charge de deux patientes atteintes de pycnodysostose : A propos d'un cas familial}

\author{
Desmyttere $\mathbf{M}^{1,3}$, De Boutray $\mathbf{M}^{2}$, Levallois $\mathrm{B}^{1}$, Torres $\mathrm{JH}^{1}$, Fauroux $\mathrm{MA}^{1}$ \\ 1 - Service d'Odontologie, CHRU de Montpellier \\ 2 - Service de CMF et Stomatologie, CHRU de Montpellier \\ 3 - Interne DESCO \\ Introduction
}

La pycnodysostose, ou maladie de Toulouse Lautrec, est une maladie génétique lysosomale rare, de transmission autosomique récessive, classée parmi les ostéo-chondro-dysplasies (CIM10). Elle provient d'une mutation du gène codant pour la cathepsine $K$ et permettant le clivage des protéines de la matrice osseuse. Son déficit conduit à la formation d'un os dense et fragile. La pycnodysostose se manifeste par plusieurs signes cliniques dont l'ostéosclérose, la fragilité osseuse, la petite taille des patients et une acro-ostéolyse des phalanges distales (Maroteaux 1962). Les symptômes maxillo-faciaux associent notamment un défaut de croissance maxillaire et mandibulaire, un angle mandibulaire ouvert et un encombrement dentaire important. De plus, les atteintes buccales de cette maladie rendent l'hygiène buccale difficile et facilitent l'apparition de lésions carieuses et de parodontopathies. Les complications attendues sont les ostéomyélites mandibulaires et les fractures pathologiques. Le diagnostic différentiel doit être établi avec l'ostéopétrose et la dysplasie cléido-crânienne. Ce travail expose le cas de 2 sœurs atteintes de pycnodysostose dans le cadre d'une pycnodysostose familiale (mère et tante maternelle également porteuses).

\section{Observations}

Une patiente de 24 ans, atteinte de pycnodysostose a consulté en novembre 2013 au Service d'Odontologie du CHRU de Montpellier. La patiente souhaitait un bilan bucco-dentaire par crainte d'un " problème dentaire grave » dans un contexte de pycnodysostose diagnostiquée dans l'enfance. L'examen clinique et radiographique a objectivé un faciès caractéristique de la maladie (avec angle mandibulaire ouvert), un encombrement dentaire ainsi que de multiples lésions carieuses et la persistance sur l'arcade de dents lactéales. Des avulsions et des soins conservateurs ont été pratiqués sous anesthésie générale, du fait de la grande anxiété de la patiente. La patiente a été revue en contrôle tous les 6 mois et ne présente à ce jour aucune complication.

La sœur de la patiente précédente, âgée de 27 ans, a consulté dans le Service en juin 2015. La patiente, atteinte de pycnodysostose, diagnostiquée à l'âge de 9 mois, se plaignait d'une tuméfaction mandibulaire droite douloureuse. L'examen endobuccal a permis d'objectiver des fistules en vestibulaire et en lingual de la dent 46, mobile. La patiente a bénéficié d'une antibiothérapie. La tomodensitométrie maxillo-faciale réalisée ensuite a objectivé une zone d'ostéolyse du secteur 4, s'étalant sur $3 \mathrm{~cm}$ le long des corticales basilaires, vestibulaires et 
linguales. Un diagnostic d'ostéomyélite mandibulaire a été posé. L'exérèse du bloc osseux incluant les dents 45 et 46 a été menée, ainsi que des biopsies osseuses multiples et étagées. Aucun germe spécifique n'a été retrouvé. Des douleurs osseuses ont été signalées par la patiente 3 mois après dans le secteur mandibulaire gauche.

Discussion et Conclusion

Ce cas illustre bien la difficulté de prise en charge des patients atteints de pycnodysostose, d'autant que leur traitement peut s'avérer lourd, associant séquestrectomie, curetage osseux voire résection mandibulaire interruptrice avec lambeau, ou encore greffe osseuse (Fenelon, 2015). Un suivi régulier chez le chirurgien-dentiste est donc nécessaire afin de réaliser les soins au plus tôt. 